\title{
Bisphenol A in Odontology: Recent data
}

\author{
Vautherin T and Lopes Cardoso I* \\ Health Sciences Faculty, University Fernando Pessoa, Porto, Portugal
}

\begin{abstract}
Background and Objective: Bisphenol A (BPA) is an organic compound of the aromatic family used mainly as a monomer, for more than 50 years in the production of polycarbonate plastics and epoxy resins. Nowadays, bisphenol A is a worrying substance that deserves special attention. The goal of this work is to focus on bisphenol A and its biological effects on the human body.

Methods: A bibliographic search was performed in databases such as PubMed, b-On and Elsevier. The keywords used were: "Bisphenol A", "BPA", "Endocrin Disruptor Chemicals".

Results: There are several known effects on the human body resulting from the exposure to BPA such as in the reproductive system, dentition, brain, behavior, cardiovascular and in immune systems. In the oral cavity, dental composites release bis-GMA and BPA immediately after placement but released doses are usually too low to lead to biological effects.
\end{abstract}

Conclusion: There are other materials such as silorans, Ormocer $^{\circledR}$ and ionomer glass cements that can be good alternatives. Clinical studies are still neaded to prove that these materials are better than the composites, being these ones still the most used materials in conservative dental medicine.

\section{Introduction}

Bisphenol A (BPA) is an organic compound belonging to the aromatic family used for more than 50 years, mainly as a monomer, in the production of polycarbonate plastics and epoxy resins. Polycarbonates are included in many everyday objects such as plastic baby bottles or bottles. On the other hand, epoxy resins are mainly found in the inner coating of cans and in composite resins.

For some years now, there has been much controversy regarding the biological effects of BPA. The first harmful effects of BPA use, were first observed in 1987 by Anna Soto and Carlos Sonnenschein in an experiment with tumor cell inhibitors. This study raised the possible role of BPA as a tumor cell initiator [1]. Its role as an endocrine disrupter had long been demonstrated [2]. However, recent studies have shown that the effects of BPA are not only hormonal but of various kinds. As a result, doubts about the effects of BPA led Canada to ban BPA bottles on $18^{\text {th }}$ October 2008. Since 2011, by European Union directive, the use of BPA has been prohibited in baby products. In 2017, the European Chemicals Agency (ECHA) classified BPA as a "matter of utmost concern".

With regard to dentistry, the problem focuses on composite resins as they are made from BPA. In addition, the aesthetic expectations of patients have been increasing and consequently the use of composite resins as well. What materials will replace composite resins in the future?

The aim of this work is to focus on bisphenol A in chemical and biological terms, as well as the legislation on its use. Next, the biological effects on the human body will be addressed. Finally, the influence of BPA on composite resins and other materials will be analyzed to evaluate possible alternatives.

A bibliographic search was performed in databases such as PubMed, b-On and Elsevier. The keywords used were: "Bisphenol A", "BPA", "Endocrin Disruptor Chemicals". The scientific articles and bibliographic reviews found were chosen from 2005 to 2019. Articles prior to this period were also chosen for their historical importance and relevance to the study. The exclusion criteria used were as follows: article whose scientific rigor would not have relevance or interest for the accomplishment of the work. Inclusion criteria were articles written in English, Portuguese or French. A total of 45 papers, books and web pages were selected with information considered relevant to this work.

\section{Bisphenol A}

\section{Chemical structure}

BPA has the chemical formula $\mathrm{C}_{15} \mathrm{H}_{16} \mathrm{O}_{2}$, being also called 4,4'-(propane-2-ylidene) diphenol or $\mathrm{p}, \mathrm{p}^{\prime}$-isopropylidenebisphenol. This compound was first synthesized in 1891 by the Russian chemist Alexandre Dianine [3].

BPA is a white solid presented as a powder or crystals. It consists of two aromatic rings linked together by a carbon bridge. This molecule is industrially synthesized from phenol and acetone by a condensation reaction catalyzed by a strong acid (usually hydrochloric acid) in the laboratory by a polystyrene base or resin (Figure 1).

\section{BPA applications}

BPA is mainly used as a monomer in the manufacture of polycarbonate plastic and epoxy resins. Polycarbonates are widely used

${ }^{\star}$ Correspondence to: Inês Lopes Cardoso, Faculdade de Ciências da Saúde, Universidade Fernando Pessoa, Rua Carlos da Maia, 296, 4200-150 Porto, Portugal, Tel: +351 225071300; E-mail: mic@ufp.edu.pt

Key words: bisphenol A, endocrin disruptor, BPA, dental composites

Received: September 20, 2019; Accepted: September 30, 2019; Published: October 03, 2019 


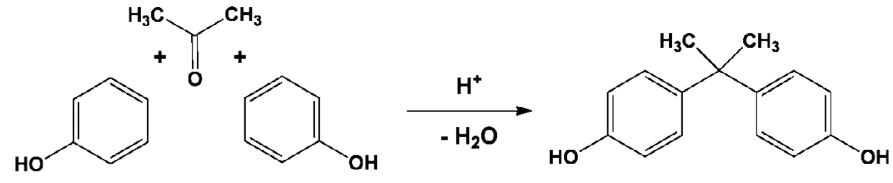

Figure 1. Reaction of bisphenol A synthesis

in everyday products such as sunglasses, CDs and food containers. In the late twentieth century, it is abundantly present in receipts (thermal paper). Some monomers are part of composite resins. BPA gives high resistance to heat and shock to plastics and is therefore widely used in construction and in the automobile industry.

\section{Dose/Effect and Legislation}

Bisphenol A is nowadays a matter of concern and therefore deserves special attention. The European Food Safety Authority (EFSA) issues a science-based warning to risk managers regarding BPA's safety when used in materials of food containers. EFSA establishes an Acceptable Daily Intake (ADI) which is the dose that can be taken daily for a lifetime without health risks. This is expressed in milligrams or micrograms per kilogram body weight per day (mg/kg bw/day). In 2006, EFSA set the ADI at $0.05 \mathrm{mg} / \mathrm{kg}$ bw/day. This daily dose was calculated from the lowest dose required for development of an effect $(50 \mathrm{mg} / \mathrm{kg}$ bw/day; LOAEL - Low Observed Adverse Effect Level) [4]. For the calculation of the daily allowable dose, without causing adverse effects, a factor of 1000 was chosen, obtaining the dose of $50 \mu \mathrm{g} / \mathrm{kg}$ bw/day, which is called NOAEL (No Observed Adverse Effect Level) [4]. In 2008, EFSA, concerned about the ability of babies and adults to eliminate BPA from the body, stated that BPA exposure should be much lower than ADI exposure. Children and adults are able to metabolize and eliminate this substance at rates much higher than DDT. In 2015, EFSA's in-depth assessment of BPA exposure and toxicity concluded that bisphenol A does not pose a risk to consumers (including newborns, infants, children and adolescents) at current exposure levels. Moreover, the ADI was changed to $0.4 \mu \mathrm{g} / \mathrm{kg}$ bw/day [5].

It has long been thought that the dose provokes the effect, that is, the higher the dose, the greater the effect. However, several years ago, some studies showed a nonlinear U-shape curve in the doseeffect relationship of BPA exposure [6]. This means that the effects are significant for low doses, decrease with increasing BPA, and increase again for high doses of this compound [6].

\section{BPA as an endocrine disruptor}

The endocrine system is one of the body's regulatory systems that works synergistically with the nervous system. This system consists of endocrine glands (pituitary, epiphysis, thyroid, parathyroid and adrenal) and endocrine cells present in organs with other functions (pancreas, gonads, kidneys and hypothalamus). Its main role is the maintenance of homeostasis and adaptation to the external environment: electrolyte and glucose balance, growth, lactation, hematopoiesis through the secretion of hormones into the blood (such as growth hormone, prolactin and insulin).

According to the World Health Organization (WHO), an endocrine disruptor is an exogenous substance or mixture of substances capable of inducing endocrine disruption in an intact organism, its descendants or subpopulations [7]. An Endocrin Disruptor Chemical (EDC) is an exogenous chemical or mixture of chemicals, that can interfere with any aspect of hormonal action. To assess the potential of EDC in the development of deleterious effects, consideration should be given to the regulation of its synthesis, secretion and hormonal actions, and the variation in regulation of these events throughout the life cycle [8].

\section{Mechanism of action and elimination}

BPA's main gateway into the body is through absorption after ingestion of contaminated food. However, BPA may enter via other routes such as absorption by the sublingual mucosa, by the skin after contact with the drug or by the lungs through contaminated air.

Historically, BPA is a synthetic estrogen and therefore has estrogenic power. This agent will act on estrogen receptors ER $\alpha$ and $\operatorname{Er} \beta$ as an agonist or antagonist depending on the situation [9]. However, its affinity for binding to those receptors is poor [10]. For this reason, it can be thought that the effects of BPA are negligible, however it can act on other receptors and produce more effects.

BPA can act on nuclear receptors - genomic pathway. BPA has been shown to have an effect on androgen [11], progesterone [11], pregnane X receptors [12], and LXR (liver X receptor) [3]. After BPAreceptor binding, this receptor becomes active triggering diverse changes. For example, androgen receptors play a very important role in physiological processes such as male embryo development and differentiation, spermatogenesis, endocrine system or mammary gland development in women [11]. LXR is implicated in this process but also in brain function, skin development, immunity and glucose homeostasis [3].

Moreover, BPA can act in the non-genomic pathway. This is much faster than the genomic pathway and can function in parallel [13]. This substance allows signal transduction by activation of receptors located in the plasma membrane, causing a high level of signal amplification, that is: a low concentration of this molecule activates this pathway and induces important modifications such as cell proliferation and differentiation or apoptosis [13]. The signaling mechanism involves movement of ions (calcium, potassium, sodium and hydrogen) that will trigger the cellular response. For example, BPA may cause calcium flux-induced prolactin secretion [14].

BPA acts on nuclear receptors by disrupting the action and regulation of hormones, such as progesterone and estrogens, by altering their synthesis, activity and inhibition mechanism [15]. It also disturbs the pituitary and thyroid hormones (T3 and T4) [16], androgens [16] and testosterone.

The major route of elimination of BPA involves its conjugation with glucuronic acid to BPA-glucuronide (BPA-G) which occurs mainly in the liver and to a lesser extent in the intestine. This reaction is catalyzed by the enzyme glucuronyltransferase, called UGT2B1 in rats while in humans there are two isoforms UGT2B15 and UGT2B7 [17]. In humans, the plasma peak of BPA-G is at 80 minutes, and its plasma concentration decreases exponentially with a half-life of 89 minutes [18]. A small part of BPA is conjugated with sulphate (BPA-S).

BPA-G and BPA-S represent the forms of elimination of BPA since these molecules are not capable of binding to estrogen receptors. However, deconugation may occur at some specific sites by the enzymes $\beta$-glucuronidase and arylsulfatase C, and BPA is free and active and may act on estrogen receptors again [19].

\section{Effects of bisphenol exposure}

Studying the effects of BPA on the human population is not easy, as for ethical reasons it is not possible to directly test the effect of BPA on humans. For this reason, studies in the human population are epidemiological, determining the concentrations of BPA to which individuals were exposed and comparing with observed pathologies. It is not possible to establish a causal relationship, but only to draw probabilistic conclusions. 
For these reasons, animal studies have been performed, although their pathophysiology is different from that of humans, and the results cannot be directly extrapolated. In addition, BPA is universally widespread and therefore difficult to have control groups of individuals not exposed to this molecule [20].

There are several known effects on human organism from exposure to BPA but it is also of great concern the possibility of absorption of this compound by the fetus since the embryonic period is critical in the development of the fetus. Several studies have shown the presence of BPA in the fetus and amniotic fluid [21]. Thus, BPA is capable of crossing the placenta [21]. Moreover, in the fetus / embryo the liver is still immature and therefore BPA metabolism is lower than in adults [21].

\section{Reproductive system}

BPA has been found to alter the synthesis and metabolism of endogenous hormones and estrogen $\alpha$ and $\beta$ receptors [22].

In men, BPA has harmful effects on the male reproductive system, disturbing its development and may even modify its structure. This compound is also toxic to the testicles, decreasing fertility by reducing sperm production, quality and mobility and increasing prostate size [23]. These changes result from the fact that gametogenesis and steroidogenesis are sensitive to estrogens.

BPA also structurally modifies the female reproductive tract. It leads to a decrease in ovarian and uterine weight, increases the occurrence of ovarian cysts and endometrial hyperplasias. The vagina also has a weight reduction, an increase in the endometrium lamina propria and endometrium estrogen and progesterone receptors [23].

Al-Hiyasat, et al. [24] were interested in the consequences of bisGMA release on rat fertility. This study showed a strong decrease in fertility in male rats, with reduced sperm count and reduced fertility rate in females who were in contact with males exposed to bis-GMA. These authors state that bis-GMA has a reprotoxic effect [24]. However, the doses of bis-GMA released by the composites are too low to induce such changes in humans.

\section{Dentition}

Exposure to BPA leads to molar incisor hypomineralization (MIH). It is a structural failure of the enamel, at least in a permanent first molar or not associated with other teeth (in most cases with an incisor). This change is characterized by porous hypomineralized enamel, with the appearance of white or yellowish / brown spots and more sensitive to fracture. This is a defect in the quality of enamel [25].

Studies have been conducted to assess the impact of BPA exposure on teeth. An increase in albumin, an increase in mRNA encoding enamelin and consequently in this protein, and a decrease in kallikrein 4 (KLK4) mRNA, a protease that degrades enamel proteins during the maturation phase [26], has been observed.

In addition, studies in rats treated for 100 days with BPA showed that they had normal erupting enamel while rats treated for 30 days had MIH-like enamel hypomineralizations [27]. This means that amelogenesis is only sensitive to MIH causative agents during a developmental exposure window. Thus, in humans this critical window would be between birth and 5 months of age because it corresponds to the mineralization period of the affected teeth in cases of MIH (incisors and first molars). This is supported by the fact that during this period the newborn is particularly sensitive to BPA due to its immature liver [27].
Thus, MIH can function as a marker for cases of early exposure to BPA [27]. In another study by the same authors, BPA has been shown to have more effect alone than associated with other endocrine disruptors such as genistein and vinclozoline [28].

\section{Brain}

Since BPA can cross the blood-brain barrier, exposure to BPA has been shown to be associated with multiple neuropsychological disorders and neurobehavioral disorders. The mechanism of action is still uncertain.

Studies using human cortical neurons (hCN) derived from human embryonic stem cells as a cellular model have been performed to study the undesirable neurotoxic effects of BPA [29]. The results revealed that chronic exposure to BPA impairs neural morphology, induces neuronal apoptosis and decreases MAP2 gene expression (neural marker) [29]. After exposure to BPA, intracellular calcium levels were elevated [29]. Therefore, BPA appears to have neurotoxic effects on these cells, causing apoptosis that may result from a disturbance of intracellular calcium homeostasis and consequent cellular dysfunction [29].

It is further described that BPA can increase aggressiveness [30], hyperactivity [31], anxiety [32], increase the risk of depression and learning disabilities [31].

This compound may also diminish the parental and social relationship. Animals exposed to BPA are found to care less for their young and play less [33].

\section{Cardiovascular system}

Many studies have been done to evaluate the relationship between BPA concentration and the risk of heart disease. In fact, in different patient groups, an increase in BPA concentration is associated with hypertension [34], decreased heart rate [34], peripheral arterial problems, and changes in the expression of genes involved in the pathophysiology of the heart.

\section{Immune system}

It has been observed that BPA appears to have proinflammatory activity, increasing some inflammation mediators such as TNF- $\alpha$, IL-8 and IL-13, and decreasing the number of macrophages [35]. EFSA claims to have new data on the effect of BPA on the immune system of animals, however there is insufficient evidence to draw conclusions about the effect on humans [36].

\section{Bisphenol A in composite resins and other materials}

\section{Composite resins}

The aesthetic and electrochemical problems of silver amalgams and the lack of biocompatibility of silicates and polymethyl methacrylates (PMMA) led to the development of a new material in the 1960s: composite resins. silane.

Composite resins consist of organic matrix, filler particles and

The organic matrix represents between 30 and $50 \%$ of the total volume and allows to have a plastic material before polymerization and hard after polymerization. In addition, the die ensures cohesion with the other elements and protects the structure against abrasion and chemical or mechanical aggression. The most commonly used matrix is bisphenol A-glycidyl methacrylate (bis-GMA or Bowen matrix). In 
addition, it is also found in diluents and viscosity controllers such as bisphenol A dimethacrylate (bis-DMA).

The mineral particles of inorganic charge, are linked to the organic matrix through silane and allow to increase the mechanical properties such as tensile strength, bending and compression and radio opacity. They also compensate for the coefficient of thermal expansion that is too high in the matrix phase.

Silane, a bipolar organo-mineral molecule, that ensures cohesion between mineral fillers and the organic matrix. This silane binds to the hydroxide groups of the fillers and polymerizes with the monomer to form a covalent bond.

Composite resins when placed in the mouth will be subject to temperature fluctuations, mechanical forces resulting from chewing or even saliva that is in permanent contact with the composite. Some researchers have shown that saliva contains cholesterol esterases that can hydrolyze bis-GMA molecules [37]. Other authors report that salivary esterases may cleave the ester binding of bis-DMA molecules, leading to BPA release [38]. The potential release of BPA from the composites used was studied to maintain retention after orthodontic treatment. These studies showed that there is a sharp increase in BPA levels in saliva 1 hour after exposure to BPA, the next day the samples already show BPA levels similar to the control. Thus, BPA release is significant within the first minutes or hours after composite placement [39].

Other studies have attempted to assess the consequences of BPA release by analyzing the effects of bis-GMA on human lymphocyte DNA [40]. The results showed that bis-GMA can damage DNA even leading to breaks in its double strand. These DNA lesions are found to be irreversible [40].

The degree of toxicity also varies with the type of composite. Flow composites have less charge and therefore more monomers. Both conventional and Flow composites will release bis-GMA, TEGDMA, UDMA and even BPA, however there is greater cytotoxicity in Flow composites compared to conventional ones [24].

Moreover, also the time used in composite polymerization appears to affect the release of BPA and therefore its toxicity. This curing time may vary between 20 and 40 seconds depending on the manufacturer. Studies have shown that a 40 second polymerization is insufficient to prevent the release of many monomers [41]. In fact, oxygen from air is a polymerization inhibitor of composites. Monomer free radicals react with oxygen in the air affecting polymerization, with only a thin layer of bis-GMA oligomers $(50-100 \mu \mathrm{m})$ forming on the surface of the composites. Therefore, unpolymerized monomers are left that will release BPA molecules into the oral cavity in the short term. It is therefore very important to follow the manufacturer's polymerization instructions.

\section{Alternative materials}

Several researches have been conducted to change the composition of composites and especially the load. To this end, these studies attempt to modify the composition of composites by introducing glass fibers, titanium dioxide nanoparticles, calcium fluorides or even antimicrobial agents $[42,43]$.

It is known that it is mainly free monomers present in the organic matrix that are most likely to cause cellular damage. For this reason, researchers have tried to incorporate new monomers into resins. These include many methacrylate derivatives, as well as new families, many of which are still experimental, such as epoxy resins, oxiranes/sylorans or spiro-ortho-carbonates (SOC) (Table 1).

Sylorans : 3M ESPE sells a silorane-based composite resin (a molecule obtained by combining a siloxane and an oxirane) whose polymerization process is quite different from that of other composite families. This composite does not have BPA in its composition, but the adhesive "Silorane System Adhesive Self-Etch" has BPA in its pure form [44]. This composite is preferably used in Class I and II posterior tooth restorations. Although oxiranes are known to be biocompatible and siloranes are non-toxic, there are still few public health studies regarding the biocompatibility of composite resins and therefore dentists often do not use these new monomers.

Ormocer $^{\circledR}$ : The main cause of failure of composite resin coatings comes from resin volume variations over time. In order to limit this disadvantage, new matrices such as the Ormocer matrices have been developed. This term is short for «Organically Modified Ceramics».

These matrices contain polymers derived from the addition of small monomer units whose nucleus consists essentially of silicon atoms associated with silanized inorganic fillers. Polysiloxane chains formed by the silicon and oxygen chain are associated with other chains that react during polymerization and contribute to an organic functionalization of the initially formed network.

Ormocers matrices combine properties of silicone, polymer hardness and thermal stability of ceramics. Compared to conventional composites, Ormocer-based dental materials have greater biocompatibility, less polymerization shrinkage and fewer residual monomers. It also has good abrasion resistance.

All of these characteristics that these organic-inorganic hybrid polymers possess seem to be very interesting in conservative dentistry. However, in this technology used in the Admira range, developed by VOCO (manufacturer), the inorganic part consists of Bis-GMA.

Studies have shown that Ormocer ${ }^{\oplus}$ matrices have properties superior to those of a standard hybrid composite and better polymerization, consequently having fewer residual monomers [45]. However, clinical confirmation is still lacking, and other studies are needed to be able to state that Ormocer ${ }^{\circledR}$ is a better product than conventional composites. Clinical studies with Ormocer ${ }^{\oplus}$ showed no significant differences from conventional composites [46] (Table 2).

Table 1. Monomers classification [43]

\begin{tabular}{|l|l|l|}
\hline Family of monomers & $\begin{array}{l}\text { Monomers in } \\
\text { comercialized products }\end{array}$ & Experimental monomers \\
\hline Methacrylate derivatives & $\begin{array}{l}\text { Bis-GMA, TEGDMA, } \\
\text { UEDMA, Bis-EMA }\end{array}$ & $\begin{array}{l}\text { DMBis-GMa, TMBis-GMA, } \\
\text { TTEMA, POSS6MA, MEP/ } \\
\text { MAA, BTDMA }\end{array}$ \\
\hline Epoxy resins & - & Imino ethylene \\
\hline Oxiranes/Sylorans & Sylorans & Oxiranes \\
\hline SOC & - & \\
\hline
\end{tabular}

Table 2. Comparison of properties of hybrid composites and composites based on Ormocer $^{\mathbb{B}}[45]$

\begin{tabular}{|l|c|c|}
\hline Mechanical properties & Hybrid composite & Ormocer \\
\hline Polimerization & Low & High \\
\hline Residual free monomers & High & Low \\
\hline Hardening & Low & High \\
\hline Flexural strength & Low & High \\
\hline Compressive strength & High & Low \\
\hline Marginal adaptation & Low & High \\
\hline
\end{tabular}


Ionomer Glass Cements (IGC): IGC allow the replacement of silicates used in previous sectors that have several disadvantages (low biocompatibility, high coefficient of thermal expansion, poor mechanical properties).

IGCs consist of a powder, an aluminum fluorine silicate, and a liquid, an aqueous solution acidifyed with maleic acid, a tricarboxylic acid used to control viscosity [47]. They have low mechanical properties and therefore, are not indicated in cases of restoration in sectors with many occlusal loads and cannot be used as a definitive material.

There are also Resin Addition Modified Ionomer Glass Cements. The incorporated resin particles are molecules like HEMA, bis-GMA that will increase their mechanical properties, however BPA is again present.

\section{Discussion}

Several biological effects of bisphenol A are now known. Several studies have raised concerns that this molecule may, in addition to other effects, cause hormonal disorders. Effects on other organs such as the brain, cardiovascular system, immune system, thyroid and reproductive system were also highlighted.

Recently, a relationship between exposure to BPA and incisormolar hypomineralization has been discovered. In response to this finding, steps have already been taken, particularly in the European Union, to limit the presence of BPA, especially in the agri-food sector, where its use is widespread.

Regarding dental composites, studies have shown that BPA is released after placement in the oral cavity. However, the released doses are low and can only be detected within hours of the application of the composites.

A direct link between BPA and changes in organism has not been demonstrated yet, but suspicions remain. Therefore, further studies, especially in humans, are needed to extrapolate experimental data to clinical practice.

\section{Conclusion}

The challenges for patients, dentists and the industry, are great. Indeed, the NINAMATA convention held in Geneva in January 2013 recommended, for environmental protection, a reduction in the use of amalgam without, for the time being, banning its use.

It has been found that the use of amalgam has been declining for several years, being replaced by composites. However, recent data on bisphenol A present in dental composites have cast doubt on the consequences it may have.

Currently, manufacturers are not yet able to provide a satisfactory material to replace composites. The BPA replacement molecule has not yet been discovered.

Even though there are some other products that can be used for preservative care for some years, such as Glass Ionomer Cements or Ormocer ${ }^{\circledR}$, they do not always meet the therapeutic requirements.

Research on new molecules remains an extremely important challenge, which should lead to the development of perfectly biocompatible new materials in the coming years.

\section{References}

1. Soto AM, Sonnenschein C (1987) Cell proliferation of estrogen-sensitive cells: the case for negative control. Endocr Rev 8: 44-52. [Crossref]
2. Dodds EC, Lawson W (1936) Synthetic estrogens without the phenanthrene nucleus. Nature 137:996.

3. Rouiller-Fabre V, Guerquin MJ, Nâ€TMTumba-Byn T, Muczynski V, Moison D, et al. (2015) Nuclear receptors and endocrine disruptors in fetal and neonatal testes: a gapped landscape. Front Endocrinol 6:1-13.

4. Vandenberg L, Ehrlich S, Belcher SM, Ben-Jonathan N, Dolinoy DC, et al. (2013) Low dose effects of bisphenol A: an integrated review of in vitro, laboratory animal, and epidemiology studies. Endocr Disruptors 1: e26490.

5. Vainio H (2019) Public health and evidence-informed policy-making: The case of a commonly used herbicide. Scand J Work Environ Health. [Crossref]

6. Villar-Pazos S, Martinez-Pinna $\mathrm{J}$, Castellano-Mũ̃ $\pm \mathrm{oz} \mathrm{M}$, Alonso-Magdalena $\mathrm{P}$, Marroqui L, et al. (2017) Molecular mechanisms involved in the non-monotonic effect of bisphenol-a on Ca2+ entry in mouse pancreatic $\hat{I}^{2}$-cells. Sci Rep 7: 1-15.

7. https://www.who.int/fr

8. Zoeller RT, Brown TR, Doan LL, Gore AC, Skakkebaek NE, et al. (2012) Endocrinedisrupting chemicals and public health protection: A statement of principles from the Endocrine Society. Endocrinology 153: 4097-4110.

9. Li Y, Burns KA, Arao Y, Luh CJ, Korach KS (2012) Differential estrogenic actions of endocrine-disrupting chemicals bisphenol A, bisphenol AF, and zearalenone through estrogen receptor $\hat{\mathrm{I}} \pm$ and $\hat{\mathrm{I}}^{2}$ in vitro. Environ Health Perspect 120: 1029-1035.

10. Blair R, Fang H, Branham WS, Hass BS, Dial SL et al. (2000) The estrogen receptor relative binding affinities of 188 natural and xenochemicals: structural diversity of ligands. Toxicol Sci 54:138-153.

11. Rehan M, Ahmad E, Sheikh IA, Abuzenadah AM, Damanhouri GA, et al. (2015) Androgen and progesterone receptors are targets for bisphenol a (BPA), 4-methyl-2,4bis-(p-hydroxyphenyl)pent-1-ene-A potent metabolite of BPA, and 4-tert-octylphenol: A computational insight. PLoS One 10: 1-18.

12. Sui Y, Ai N, Park SH, Rios-Pilier J, Perkins JT, et al. (2012) Bisphenol A and its analogues activate human pregnane X receptor. Environ Health Perspect 120: 399-405.

13. Viñas R, Jeng YJ, Watson CS (2012) Non-genomic effects of xenoestrogen mixtures Int J Environ Res Public Health 9: 2694-2714. [Crossref]

14. Wozniak A, Bulayeva N, Watson S (2005) Xenoestrogens at picomolar to nanomolar concentrations trigger membrane estrogen receptor- $\hat{\mathrm{I}} \pm$-mediated $\mathrm{Ca} 2+$ fluxes and prolactin release in GH3/B6 pituitary tumor. Environ Health Perspect 113: 431-439.

15. Aldad T, Rahmani N, Leranth C, Taylor HS (2011) Bisphenol-A exposure alters endometrial progesterone receptor expression in the nonhuman primate. Fertil Steril 96: $175-179$.

16. Vom Saal F, Hughes C (2005) An extensive new literature concerning low-dose effects of bisphenol A shows the need for a new risk assessment. Environ Health Perspect 113: 926-933.

17. Mazur C, Kenneke JF, Hess-Wilson JK, Lipscomb JC (2010) Differences between human and rat intestinal and hepatic bisphenol a glucuronidation and the influence of alamethicin on in vitro kinetic measurements. Drug Metab Dispos 38: 2232-2238.

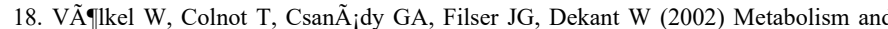
kinetics of bisphenol a in humans at low doses following oral administration. Chem Res toxicol 15: 1281-1287.

19. Ginsberg G, Rice C (2009) Does rapid metabolism ensure negligible risk from bisphenol A?. Environ Health Perspect 117: 1639-1643.

20. Lang I, Galloway TS, Scarlett A, Henley WE, Depledge M, et al. (2008) Association of urinary bisphenol A concentration abnormalities in adults. JAMA 300: 1303-1310.

21. Corrales J, Kristofco LA, Steele WB, Yates BS, Breed CS, et al. (2015) Globa Assessment of Bisphenol A in the Environment: Review and Analysis of Its Occurrence and Bioaccumulation. Dose Response 13: 1559325815598308. [Crossref]

22. Sonnenschein C, Soto AM (1998) An updated review of envrionmental estrogen and androgen mimics and antagonists. J Steroid Biochem Mol Biol 65: 143-150.

23. Vandenberg LN, Maffini MV, Wadia PR, Sonnenschein C, Rubin BS, et al. (2007) Exposure to environmentally relevant doses of the xenoestrogen bisphenol-A alters development of the fetal mouse mammary gland. Endocrinology 148: 116-127.

24. Al-Hiyasat AS, Darmani H (2006) In vivo effects of BISGMA-a component of dental composite-on male mouse reproduction and fertility. J Biomed Mater Res A 78: 66-72. [Crossref]

25. Cabral RN, Nyvad B, Soviero VLVM, Freitas E4, Leal SC5 (2019) Reliability and validity of a new classification of MIH based on severity. Clin Oral Investig. [Crossref] 
26. Jedeon K, de la Dure-Molla M, Brookes SJ, Loiodice S, Marciano C, et al. (2013) Enamel defects reflect perinatal exposure to bisphenol A. Am J Pathol 183: 108-118.

27. Jedeon K, Marciano C, Loiodice S, Boudalia S, Carnivenc Lavier MC, et al. (2014) Enamel hypomineralization due to endocrine disruptors. Connect Tissue Res 55: 43-47.

28. Jedeon K, Houari S (2016) Chronic Exposure to Bisphenol A Exacerbates Dental Fluorosis in Growing Rats. J Bone Miner Res 31: 1955-1966. [Crossref]

29. Wang H, Zhao P, Huang Q, Chi Y, Dong S, et al. (2019) Bisphenol-A induces neurodegeneration through disturbance of intracellular calcium homeostasis in human embryonic stem cells-derived cortical neurons. Chemosphere 229: 618-630.

30. Kundakovic M, Champagne A (2011) Epigenetic perspective on the developmental effects of bisphenol A. Brain Behav Immun 25: 1084-1093.

31. Saili KS, Corvi MM, Weber DN, Patel AU, Das SR, et al. (2012) Neurodevelopmental low-dose bisphenol A exposure leads to early life-stage hyperactivity and learning deficits in adult zebrafish. Toxicology 291: 83-92.

32. JasareviÄł E, Williams SA, Vandas GM, Ellersieck MR, Liao C, et al. (2013) Sex and dose-dependent effects of developmental exposure to bisphenol A on anxiety and spatial learning in deer mice (Peromyscus maniculatus bairdii) offspring. Horm Behav 63: 180-189.

33. Weber DN, Hoffmann RG, Hoke ES, Tanguay RL (2015) Bisphenol a exposure during early development induces sex-specific changes in adult zebrafish social interactions. $J$ Toxicol Environ Health A 78: 50-66.

34. Bae S, Kim JH, Lim YH, Park HY, Hong YC (2012) Associations of bisphenol A exposure with heart rate variability and blood pressure. Hypertension 60: 786-793. [Crossref]

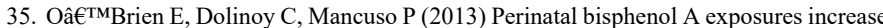
production of pro-inflammatory mediators in bone marrow-derived mast cells of adult mice. J Immunotoxicol 11: 205-212.

36. Vainio H (2019) Public health and evidence-informed policy-making: The case of a commonly used herbicide. Scand J Work Environ Health. [Crossref]
37. Gitalis R, Zhou L, Marashdeh MQ, Sun C, Glogauer M, et al. (2019) Human neutrophils degrade methacrylate resin composites and tooth dentin. Acta Biomater 88: 325-331.

38. Söderholm KJ, Mariotti A (1999) BIS-GMA--based resins in dentistry: are they safe? $J$ Am Dent Assoc 130: 201-209. [Crossref]

39. Moreira M, Matos LG, de Souza ID, Brigante TA, Queiroz ME, et al. (2017) Bisphenol A release from orthodontic adhesives measured in vitro and in vivo with gas chromatography. Am J Orthod Dentofacial Orthop 151: 477-483.

40. Drozdz K, Wysokinski D, Krupa R, Wozniak K (2011) Bisphenol A-glycidyl methacrylate induces a broad spectrum of DNA damage in human lymphocytes. Arch Toxicol 85: 1453-1461.

41. Polydorou O, Trittler R, Hellwig E, Kümmerer K (2007) Elution of monomers from two conventional dental composite materials. Dent Mater 23: 1535-1541. [Crossref]

42. Ferracane JL (2011) Resin composite--state of the art. Dent Mater 27: 29-38. [Crossref]

43. Berthault G, Durand AL, Lasfargues JJ, Decup F (2008) Les nouveaux compositesâ $€^{-}$: $\tilde{A} \odot v a l u a t i o n$ et int $\tilde{A} \odot \mathrm{r} \tilde{A}^{a}$ ts cliniques pour les restaurations en technique directe. $R e v$ Odont Stomat 37: 177-197.

44. Weinmann W, Thalacker C, Guggenberger R (2005) Siloranes in dental composites. Dent Mater 21: 68-74. [Crossref]

45. Kalra S, Singh A, Gupta M, Chadha V (2012) Ormocer: An aesthetic direct restorative material; An in vitro study comparing the marginal sealing ability of organically modified ceramics and a hybrid composite using an ormocer-based bonding agent and a conventional fifth-generation bonding agent. Contemp Clin Dent 3: 4853.

46. Zimmerli B, Strub M, Jeger F, Stadler O, Lussi A (2010) Composite materials: composition, properties and clinical applications. Schweiz Monatsschr Zanmed 120 972-979.

47. Kovarik RE, Haubenreich JE, Gore D (2005) Glass ionomer cements: a review of composition, chemistry, and biocompatibility as a dental and medical implant material $J$ Long Term Eff Med Implants 15: 655-671.

Copyright: $₫ 2019$ Vautherin T. This is an open-access article distributed under the terms of the Creative Commons Attribution License, which permits unrestricted use, distribution, and reproduction in any medium, provided the original author and source are credited. 